\title{
Diagnosis of immunodeficiency caused by a purine nucleoside phosphorylase defect by using tandem mass spectrometry on dried blood spots
}

\author{
Giancarlo la Marca, PharmSc, ${ }^{a, b *}$ Clementina Canessa, MD, ${ }^{c, d}$ Elisa Giocaliere, BSc, ${ }^{a, b}$ Francesca Romano, BSc, $^{\text {c,d }}$ \\ Sabrina Malvagia, BSc, ${ }^{\text {a,b }}$ Silvia Funghini, PhD, ${ }^{\text {a,b }}$ Maria Moriondo, BSc, ${ }^{\text {c,d }}$ Claudia Valleriani, BSc, ${ }^{\text {c,d }}$ \\ Francesca Lippi, MD, ${ }^{\text {c,d }}$ Daniela Ombrone, BSc, ${ }^{\text {a,b }}$ Maria Luisa Della Bona, PharmSc, ${ }^{\text {a,b }}$ Carsten Speckmann, MD, ${ }^{\text {e }}$

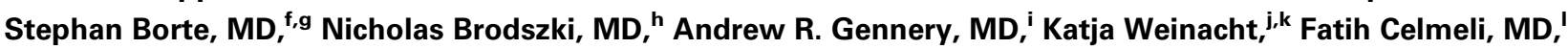

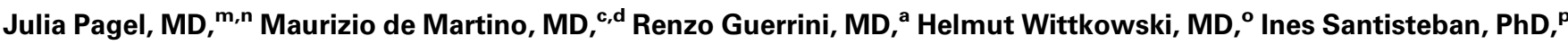 \\ Pawan Bali, PhD, ${ }^{\mathbf{p}}$ Aydan Ikinciogullari, MD, ${ }^{\mathbf{q}}$ Michael Hershfield, MD, ${ }^{\mathbf{p}}$ Luigi D. Notarangelo, MD, $^{\mathbf{r}}$ \\ Massimo Resti, MD, ${ }^{\mathbf{c}, \mathbf{d} *}$ and Chiara Azzari, MD ${ }^{\mathbf{c}, \mathbf{d} *}$ \\ Florence, Italy, Freiburg, Leipzig, Lübeck, and Münster, Germany, \\ Stockholm and Lund, Sweden, Boston, Mass, Newcastle upon Tyne, United Kingdom, Antalya and Ankara, Turkey, and Durham, NC
}

Background: Purine nucleoside phosphorylase (PNP) deficiency is a rare form of autosomal recessive combined primary immunodeficiency caused by a enzyme defect leading to the accumulation of inosine, $2^{\prime}$-deoxy-inosine (dIno), guanosine, and 2 '-deoxy-guanosine (dGuo) in all cells, especially lymphocytes. Treatments are available and curative for PNP deficiency, but

$\overline{\text { From }}{ }^{\mathrm{a}}$ Newborn Screening, Biochemistry and Pharmacology Laboratory, Paediatric Neurology Unit and Laboratories, Neuroscience Department, Meyer Children's Hospital, Florence; ${ }^{b}$ the Department of Neurosciences, Psychology, Pharmacology and Child Health, University of Florence; 'Meyer Children's University Hospital, Florence; ${ }^{d}$ the Department of Health Sciences, University of Florence; ${ }^{\mathrm{e}}$ Zentrum fur Kinderheilkunde und Jugendmedizin, Centrum fuer Chronische, ImmundefizienzUniversitaet Freiburg; ${ }^{f}$ the Division of Clinical Immunology and Transfusion Medicine, Department of Laboratory Medicine, Karolinska Institutet, Karolinska University Hospital Huddinge, Stockholm; ${ }^{\mathrm{g}}$ the Translational Centre for Regenerative Medicine (TRM), University of Leipzig; ${ }^{\text {h }}$ the Department of Allergy and Immunology,

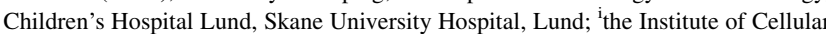
Medicine, Newcastle University; ${ }^{\mathrm{j}}$ the Division of Hematology and Oncology, Children's Hospital, Boston; ${ }^{\mathrm{k}}$ the Department of Pediatric Oncology, Dana-Farber Cancer Institute, Boston; ${ }^{1}$ Antalya Education and Research Hospital, Pediatric Immunology and Allergy, Antalya; ${ }^{\mathrm{m}}$ Pädiatrische Hämatologie und Onkologie, Universitätsklinikum Schleswig-Holstein, Campus Lübeck; ${ }^{n}$ the Department of General Pediatrics and ${ }^{\circ}$ Pediatric Rheumatology and Immunology, University Children's Hospital Muenster; ${ }^{p}$ the Department of Medicine, Duke University, Medical Center, Durham; ${ }^{\mathrm{q}}$ the Department of Pediatric Immunology and Allergy, Ankara University Medical School, Ankara; and 'the Division of Immunology, Children's Hospital, Boston.

*These authors contributed equally to this work.

Supported in part by a donation from Famiglia Cassigoli (to C.A.) and a grant from the University of Florence (to C.A. and G.1.M.), Meyer Children's University Hospital (to M.R.), and the Tuscany (Italy) region (to C.A., G.1.M., and M.R.). Sigma-Tau Pharmaceuticals provided grant support to M.H. and I.S. Orphan Europe provided grant support to E.G. and F.R.

Disclosure of potential conflict of interest: I. Santisteban and P. Bali have received research support from Sigma-Tau Pharmaceuticals. M. Hershfield has received research support, consultancy fees, and travel support from Sigma-Tau Pharmaceuticals. L. D. Notarangelo is a board member for the Program in Molecular and Cellular Medicine, Boston, and for Pediatric University Hospital "Meyer," Florence, Italy; is employed by Boston Children's Hospital; has received research support from the National Institutes of Health and the March of Dimes; and has received royalties from UpToDate. The rest of the authors declare that they have no relevant conflict of interest.

Received for publication July 15, 2013; revised December 27, 2013; accepted for publication January 3, 2014.

Available online April 24, 2014.

Corresponding author: Chiara Azzari, MD, Department of Health Sciences, University of Florence, Meyer Children's University Hospital, Viale Pieraccini 24, 50139 Firenze, Italy. E-mail: chiara.azzari@unifi.it.

0091-6749/\$36.00

(C) 2014 American Academy of Allergy, Asthma \& Immunology

http://dx.doi.org/10.1016/j.jaci.2014.01.040 their efficacy depends on the early approach. PNP-combined immunodeficiency complies with the criteria for inclusion in a newborn screening program.

Objective: This study evaluate whether mass spectrometry can identify metabolite abnormalities in dried blood spots (DBSs) from affected patients, with the final goal of individuating the disease at birth during routine newborn screening. Methods: DBS samples from 9 patients with genetically confirmed PNP-combined immunodeficiency, 10,000 DBS samples from healthy newborns, and 240 DBSs from healthy donors of different age ranges were examined. Inosine, dIno, guanosine, and dGuo were tested by using tandem mass spectrometry (TMS). T-cell receptor excision circle (TREC) and kappa-deleting recombination excision circle (KREC) levels were evaluated by using quantitative RT-PCR only for the 2 patients (patients 8 and 9) whose neonatal DBSs were available. Results: Mean levels of guanosine, inosine, dGuo, and dIno were 4.4, 133.3, 3.6, and $3.8 \mu \mathrm{mol} / \mathrm{L}$, respectively, in affected patients. No indeterminate or false-positive results were found. In patient 8 TREC levels were borderline and KREC levels were abnormal; in patient 9 TRECs were undetectable, whereas KREC levels were normal.

Conclusion: TMS is a valid method for diagnosis of PNP deficiency on DBSs of affected patients at a negligible cost. TMS identifies newborns with PNP deficiency, whereas TREC or KREC measurement alone can fail. (J Allergy Clin Immunol 2014;134:155-9.)

Key words: Purine nucleoside phosphorylase, severe combined immunodeficiency, newborn screening, tandem mass spectrometry, late-onset, delayed-onset, purine nucleoside phosphorylase-combined immunodeficiency, T-cell receptor excision circle, inherited disorder

Primary immunodeficiencies are a group of severe diseases that affect the immune system. The most severe phenotype is severe combined immunodeficiency (SCID), in which both cellular and humoral immunity are affected. ${ }^{1}$ Even though healthy at birth, infants with SCID usually die of severe infections in the first years of life unless early and effective therapy is provided. ${ }^{1}$ Usually, the diagnosis of SCID is hypothesized because of the presence of a severe infection; at that time, permanent damage can already be present. ${ }^{1}$ Therefore an early diagnosis in the preinfection period is desirable to save affected patients and to afford them good 


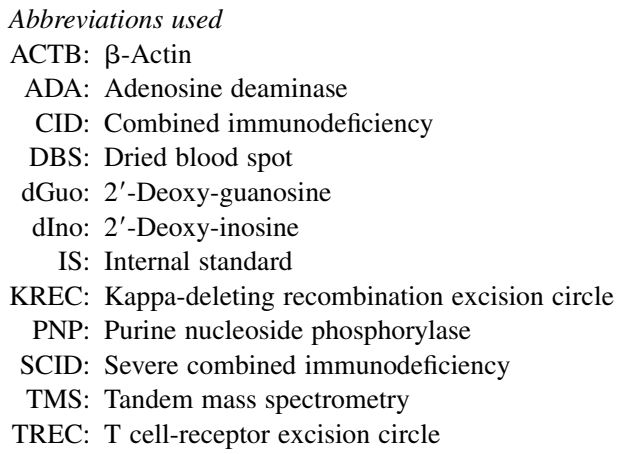

quality of life. Newborn screening of SCID has been proposed and is already performed in several countries. ${ }^{2}$ The high mortality and morbidity rates of the disease, availability of curative therapies, higher effectiveness of those therapies when performed during the early presymptomatic phase, and availability of specific disease markers makes the SCIDs a group of diseases deserving of newborn screening.

Purine nucleoside phosphorylase (PNP) deficiency is a rare form of autosomal recessive combined immunodeficiency (CID), accounting for approximately $4 \%$ of patients with $\operatorname{SCID}^{4-6}$ with a wide spectrum of immunologic severity up to PNP-SCID. Nevertheless, its true incidence and prevalence remains unknown because of misdiagnosis and insufficient available laboratory testing.

PNP is a key enzyme in the purine degradation and salvage pathway that catalyses phosphorolysis of purine nucleosides and $2^{\prime}$-deoxy-nucleosides, such as guanosine, $2^{\prime}$-deoxy-guanosine (dGuo), inosine, and 2'-deoxy-inosine (dIno), to their respective purine bases and pentose-1-phosphates. ${ }^{7}$ Loss of PNP function blocks production of xanthine, hypoxanthine, and uric acid and allows PNP substrates to undergo alternative metabolism to toxic metabolites. The intracellular accumulation of deoxyguanosine triphosphate derived from dGuo is thought to be toxic to lymphoid cells, and this or other effects of PNP deficiency result in various neurologic abnormalities in up to half of patients with the condition. $^{6,8}$

PNP deficiency is both genetically and clinically heterogeneous: inheriting alleles with mutations that profoundly decrease or abolish enzyme activity results in CID, which has a wide clinical spectrum up to SCID; less detrimental mutations confer milder phenotypes. ${ }^{8}$ PNP deficiency should be suspected in a patient with $\mathrm{T}$ lymphopenia, recurrent infections of the upper and lower respiratory tract caused by common bacterial pathogens or opportunistic organisms, neurologic abnormalities, autoimmunity, or malignancy. ${ }^{1,4-6,8}$ Hematopoietic stem cell transplantation might be curative, at least for the immunologic aspects of the disease, but its efficacy is higher if performed early to restore lymphoid cells that express active PNP and provide both immune function and metabolic detoxification to other organs before irreversible damage has occurred.

PNP deficiency is usually diagnosed in already symptomatic patients by assaying enzyme activity in hemolysates prepared from fresh or frozen packed erythrocytes, in dried blood spots (DBSs), or in blood mononuclear cells or fibroblasts. The diagnosis can also be established or confirmed by means of analysis of PNP genotype. ${ }^{8}$ We have recently demonstrated that tandem mass spectrometry (TMS) can easily identify abnormal metabolites in a similar disorder, SCID, because of inherited deficiency of adenosine deaminase (ADA). ${ }^{9}$ The specificity and sensitivity of TMS diagnosis of ADA deficiency is very high and has a very low cost when used on DBSs during a routine newborn screening procedure. ${ }^{9,10}$ Since 2010 , a pilot population-based newborn screening study for SCID has been started in Tuscany, Italy, using both $\mathrm{TMS}^{9,10}$ and molecular methods to evaluate T-cell receptor excision circles (TREC) ${ }^{11}$ and, more recently, kappa-deleting recombination excision circles (KREC) levels. ${ }^{12}$ TRECs and KRECs are well-known markers of primary immunodeficiency disorders used in newborn screening programs. TREC and KREC levels are evaluated by using quantitative real-time PCR on DBSs. However, both TREC and KREC quantitative analysis might fail in identification of some immunodeficiencies ${ }^{9}$ and cannot individuate delayed or late-onset ADA deficiency.

The aim of the present work was to evaluate whether TMS can identify PNP metabolites in DBSs, with the final goal of finding a sensitive and reliable tool able to identify patients with PNP-CID at birth during routine newborn screening.

\section{METHODS \\ Patients}

DBS samples from 9 patients with genetically confirmed PNP deficiency were evaluated. Four of them were from Turkey, 2 were from Germany, 1 was from Argentina, 1 was from the United Kingdom, and 1 was from Sweden. DBSs were taken at the time of diagnosis in 7 of these patients (patients 1-7), although for 2 patients (patients 8 and 9), stored DBSs taken at birth during routine newborn screening were retrieved and retrospectively tested. The 2 patients (patients 8 and 9) with available DBSs taken at birth came from the United Kingdom and Sweden.

All patients had severe recurrent infections, neurologic symptoms, or both. Clinical data are summarized in Table I.

\section{Carriers}

In addition to samples from affected patients, DBSs from the mother of patient 1 and both parents from patients 3 and 6 were analyzed. All of them were single-allele carriers of the mutation and clinically healthy.

\section{Guthrie cards from PNP-deficient patients, carriers, and control subjects}

All experiments for the quantitation of PNP substrates were conducted in compliance with institutional review board guidelines (protocol no. 7949/ 2011). Informed consent was obtained from parents or guardians.

As controls, 10,000 DBSs from neonates (9,300 full-term and 700 premature infants weighing $<1,800 \mathrm{~g}$ ) born in the period between January 2012 and May 2013 were analyzed; 240 DBSs from healthy subjects divided into 4 age groups were also analyzed.

DBSs were obtained from spotted blood on filter paper (903; Whatman $\mathrm{GmbH}$, Dassel, Germany) immediately after withdrawal of the samples to prevent further metabolic conversions.

\section{Guthrie card collection procedure for newborn screening}

DBS samples are routinely collected from all neonates born in the Tuscany and Umbria regions of Italy (about 45,000 samples per year). Collection is recommended at between 48 and 72 hours of life. Blood samples are obtained by means of heel sticks, spotted on filter paper (903, Whatman $\mathrm{GmbH})$, dried, and sent daily by courier to the central newborn screening laboratory. 
TABLE I. Patients' clinical data

\begin{tabular}{|c|c|c|c|c|}
\hline & Age at diagnosis & Origin & Clinical symptoms & Laboratory data \\
\hline Patient 1 & $11 \mathrm{mo}$ & Turkey & Recurrent otitis & $\begin{array}{l}\text { Lymphopenia, reduced response to mitogens, } \\
\text { low uric acid levels }\end{array}$ \\
\hline Patient 2 & $3 \mathrm{y}, 9 \mathrm{mo}$ & Turkey & $\begin{array}{l}\text { Recurrent pneumonia, motor and mental } \\
\text { retardation }\end{array}$ & $\begin{array}{l}\text { Lymphopenia, reduced response to mitogens, } \\
\text { low uric acid levels }\end{array}$ \\
\hline Patient 3 & $1 \mathrm{y}, 3 \mathrm{~m}$ & Turkey & $\begin{array}{l}\text { Frequent upper airway infections, behavioral } \\
\text { problems }\end{array}$ & $\begin{array}{l}\text { Lymphopenia, reduced response to mitogens, } \\
\text { low uric acid levels }\end{array}$ \\
\hline Patient 4 & $13 \mathrm{y}$ & Germany & Bronchiectasis, nonprogressive cerebral palsy & $\begin{array}{l}\text { Lymphopenia, reduced response to mitogens, } \\
\text { low uric acid levels }\end{array}$ \\
\hline Patient 5 & $3 \mathrm{y}, 5 \mathrm{mo}$ & Germany & $\begin{array}{l}\text { Recurrent sinusitis, autoimmune } \\
\text { thrombocytopenia, EBV-positive B-cell } \\
\text { lymphoma }\end{array}$ & $\begin{array}{l}\text { Lymphopenia, reduced response to mitogens, } \\
\text { low uric acid levels }\end{array}$ \\
\hline Patient 6 & $3 \mathrm{y}, 6 \mathrm{~m}$ & Argentina & $\begin{array}{l}\text { Recurrent otitis media and pulmonary } \\
\text { infections, bronchiectasis, spastic lower limb } \\
\text { palsy, autoimmune thrombocytopenia }\end{array}$ & Lymphopenia, low serum uric acid level \\
\hline Patient 7 & $14 \mathrm{mo}$ & Turkey & $\begin{array}{l}\text { Recurrent respiratory tract infections, truncal } \\
\text { hypotonia, failure to thrive }\end{array}$ & $\begin{array}{l}\text { Lymphopenia, reduced response to mitogens, } \\
\text { low uric acid levels }\end{array}$ \\
\hline Patient 8 & $14 \mathrm{y}$ & United Kingdom & $\begin{array}{l}\text { Developmental delay, trunk ataxia, bacterial } \\
\text { pneumonia }\end{array}$ & Reduced responses to mitogens by lymphocytes \\
\hline Patient 9 & $3 \mathrm{y}, 6 \mathrm{mo}$ & Sweden & $\begin{array}{l}\text { Veno-occlusive disease; severe pneumonitis; } \\
\text { skin, gut, and renal graft-versus-host disease } \\
\text { after HSCT; nonprogressive global } \\
\text { developmental delay }\end{array}$ & \\
\hline
\end{tabular}

An expanded newborn screening with TMS is routinely performed for more than 40 inborn errors of metabolism of amino acids, organic acids, fatty acids, and ADA-SCID. ${ }^{9}$ A pilot study in the Florence area (6000 samples per year) is also being performed in our laboratory with TREC measurement. All Guthrie cards are analyzed within 2 days from collection; after use, all cards are stored and, if not used, discarded after 10 years.

\section{Standards and chemicals}

Guanosine, inosine, dGuo, and dIno were purchased from Sigma-Aldrich (St Louis, Mo); labeled guanosine, inosine, and dGuo to be used as internal standards (ISs) were purchased from Cambridge Isotope Laboratories (Andover, Mass). Stock solutions of chemical and labeled standards at $100 \mu \mathrm{mol} / \mathrm{L}$ were prepared in HPLC-grade water and stored at $-20^{\circ} \mathrm{C}$. Working solutions were prepared daily from stock solutions. Other reagents, such as HPLC-grade water, formic acid, and acetonitrile, were purchased from Panreac (Barcelona, Spain).

\section{TMS and method validation}

A Triple-Quad Mass Spectrometer (ABI SCIEX API 4000, Toronto, Ontario, Canada) equipped with a turbo ion-spray source and coupled to an analytic HPLC device was used for this study.

The method for individuating PNP metabolites on DBS samples has been patented (PCT EP2010/070517). The method provides quantitative analysis of different metabolites, among them guanosine, dGuo, inosine, and dIno. The method has been tested with 10,000 DBSs from healthy newborns. TMS parameters and method validation details, as well as normal values, are provided in the Methods section in this article's Online Repository at www. jacionline.org. Validation was performed by using calibrators at a known concentration of PNP metabolites in terms of linearity, intraday and interday precision, and accuracy (see Table E1 in this article's Online Repository at www. jacionline.org).

Sample preparation for routine screening tests is exactly as reported by la Marca et al ${ }^{13}$ with the exception of the butylation procedure, which is not carried out.

\section{TREC and KREC analysis for patients 8 and 9}

Details on TREC and KREC analysis for patients 8 and 9 are provided in the Methods section in this article's Online Repository.

\section{PNP activity}

PNP enzyme activity was measured by using a radiochemical method ${ }^{14}$ and adapted for measuring the activity in eluates of DBSs on filter paper. ${ }^{15}$ For patient 9 , enzyme activity was evaluated on induced pluripotent stem cells.

\section{Cost analysis of TMS}

Costs for reagents, equipment, and laboratory operators have been considered to evaluate the total cost of the test per patient. Costs of Guthrie cards and transport to the newborn screening laboratory were not included because the test was performed on the same Guthrie card already used for all other neonatal screening routinely performed in Tuscany.

\section{RESULTS}

Ten thousand blood spots were tested to establish normal ranges for PNP metabolites. Moreover, 240 DBSs from healthy donors were analyzed to establish age-related cutoffs in a nonneonatal population. We decided to divide the reference population into 4 age groups.

Furthermore, we investigated whether PNP metabolite accumulation could increase the newborn screening cutoff because of enzymatic immaturity in preterm neonates. No significant differences were found by comparing the means and SDs between the full-term neonate group with those in the premature neonate group (data not shown). We defined the same reference ranges for all PNP metabolites in both groups. The reference values for dGuo, guanosine, inosine, and dIno have been summarized in Table II. As for control subjects, no false-positive or indeterminate results were found.

All the DBS samples obtained from the 9 patients with confirmed PNP-CID demonstrated high levels of inosine, dIno, guanosine, and dGuo compared with those in control subjects. In patients 1 to 7, for whom neonatal DBSs were not available, the metabolite measurement was performed on DBS samples prepared at the time when PNP deficiency was diagnosed (11 months 
TABLE II. Reference values for dGuo, guanosine, inosine, and dlno

\begin{tabular}{|c|c|c|c|c|}
\hline & $\begin{array}{c}\text { dGuo } \\
(\mu \mathrm{mol} / \mathrm{L})\end{array}$ & $\begin{array}{c}\text { Guanosine } \\
(\mu \mathrm{mol} / \mathrm{L})\end{array}$ & $\begin{array}{c}\text { Inosine } \\
(\mu \mathrm{mol} / \mathrm{L})\end{array}$ & $\begin{array}{c}\text { dlno } \\
(\mu \mathrm{mol} / \mathrm{L})\end{array}$ \\
\hline Newborns & 0.09 & 1.50 & 20.70 & 0.28 \\
\hline $1-6 \mathrm{mo}$ & 0.01 & 0.29 & 41.86 & 0.68 \\
\hline $6 \mathrm{mo}-5 \mathrm{y}$ & 0.01 & 0.29 & 21.74 & 0.33 \\
\hline $5-15 y$ & 0.01 & 0.22 & 17.92 & 0.41 \\
\hline$>15 \mathrm{y}$ & 0.01 & 0.18 & 13.85 & 0.43 \\
\hline
\end{tabular}

Values are calculated as medians +2 SDs.

to 13 years of age). Only for patients 8 and 9 was retrospective analysis performed on neonatal DBSs taken and stored in their country of origin (Table I).

In the 5 adult carriers concentrations of all metabolites within normal ranges were found.

Fig E1 in this article's Online Repository at www.jacionline. org shows levels of the 4 metabolites in 2 patients ( 1 tested at birth and the other tested at the age of diagnosis), 1 representative control subject, and a carrier.

TREC and KREC assays were performed only for patients 8 and 9, for whom neonatal DBSs were available. Levels are shown in Table III. ${ }^{16-19}$

The activities of PNP in cells from patients 1 to 8 and in induced pluripotent stem cells from patient 9 and the results from sequence analysis of the PNP gene are presented in Table III. Enzyme activity was defective in all patients, with the exception of patient 5, who had borderline values.

As previously described for ADA screening using TMS, ${ }^{9}$ no extra instruments and no extra operator time were necessary other than the time required for routine neonatal screening. Therefore the cost is calculated for reagents only and is approximately $€ 0.01$ (\$0.013) per DBS tested.

\section{DISCUSSION}

The results of the present study demonstrate that TMS can quantify purine metabolites on DBSs to identify patients with PNP deficiency. The method can identify PNP-deficient patients at birth on DBSs taken during the newborn screening procedure, thereby allowing early diagnosis and the most effective therapeutic intervention.

The sensitivity and specificity of the described method were tested by using retrospective analysis of DBSs from 9 patients with a genetically confirmed diagnosis of PNP deficiency and on a group of 10,240 DBSs from healthy control subjects. All patients with PNP-CID were identified based on PNP metabolite levels out of the normal range, even those neonatal DBSs (patients 8 and 9) in which TREC or KREC analysis results were not out of normal ranges and in 1 unique case in which a deficiency of PNP enzymatic activity in the DBS sample was not diagnostic. Actually, because there is no overlapping between metabolite levels of PNP-deficient patients and control subjects, the risk of false-positive and false-negative results is extremely low. Experimental data showed some differences in median values of agerelated metabolite concentrations (Table II). These results could be due to different enzymatic metabolism related to dietary intake, cellular turnover, or growth curve in newborns compared with older subjects.

In addition, the method is applicable to premature infants, whose mean PNP metabolite levels do not differ from those found in full-term babies. Levels of all PNP metabolites in the 5 carrier subjects were comparable with those seen in healthy control subjects. These data were not unexpected because similar results had been obtained with TMS analysis of carriers of ADA deficiency. ${ }^{9}$ Enzymatic activity had been previously tested in parents of PNP-deficient patients (Hershfield M, personal communication); it was approximately $50 \%$ of normal activity, and therefore it is not surprising that PNP metabolite levels are not increased in carriers. We could not test patients at different times of life, and therefore we cannot demonstrate in this study whether metabolite levels remain at the same level during the lifespan. Studies are now going on in our laboratories to answer this question. However, data obtained in patients with ADA deficiency, which has a similar pathogenesis, suggest that metabolite levels strongly correlate with both genetic variant and enzymatic activity and therefore tend to remain stable throughout life. $^{10}$

TMS could be a useful tool for this follow-up study because it is very easy to perform and inexpensive. No extra operator time is necessary for sample preparation or analysis other than the time required for routine neonatal screening. Similarly, no extra equipment is necessary, and instruments are not used for longer times. Therefore the cost is calculated for reagents only; including unavoidable reagent waste, the cost is $€ 0.01$ ( $\$ 0.013$ ) per test.

Moreover, TMS could be applied in the follow-up period to evaluate how PNP metabolites are affected by a treatment like hematopoietic stem cell transplantation.

It is well known that the immune defect observed in PNP-deficient patients can be heterogeneous, with predominance of either T- or B-cell defects in different patients. This aspect might reflect what we found in TREC and KREC analysis on DBSs obtained at birth. Actually, in the 2 patients whose neonatal DBSs were available, TREC and KREC levels were not concordant. Both TREC and KREC levels tended to be low in both patients, but although in patient 8 TREC levels were borderline and not reported as pathologic in the presence of absolutely abnormal KREC levels, in patient 9 TRECs were undetectable, whereas KREC levels were reported as normal. Our data suggest that, as previously demonstrated for ADA deficiency, TREC $^{10,20}$ or KREC measurement, when used alone (data not shown), can fail to identify an affected patient, whereas TMS has a higher sensitivity. However, our data on TREC and KREC efficiency in PNP diagnosis at birth should be completed in larger studies because no definitive conclusion can be drawn from the limited number of neonatal DBSs studied.

The data also suggest that the 2 tests (TREC and KREC measurement) should be used together to catch the highest number of patients with SCID. ${ }^{12}$ The combination of the TREC plus KREC assay can maximize the effect of identifying various types of patients with SCID.

The finding that dIno and dGuo levels in DBSs taken at birth range between similar values to those found in samples taken at the time of diagnosis suggests that metabolites do not progressively accumulate during life, but as similar for ADA deficiency, toxic levels of metabolites might already be present at birth. The use of TMS for PNP measurement, together with established routine newborn screening programs, would raise suspicion of PNP deficiency at an extremely early age, thereby giving the clinician the opportunity to confirm the diagnosis (by using an enzyme assay and genotyping) and to initiate the appropriate therapy in time. 
TABLE III. Purine levels, genetic analysis, and enzymatic activity in 9 patients with PNP-CID

\begin{tabular}{|c|c|c|c|c|c|c|c|c|c|c|c|}
\hline $\begin{array}{l}\text { Patient } \\
\text { no.* }\end{array}$ & $\begin{array}{c}\text { Age at DBS } \\
\text { collection }\end{array}$ & $\begin{array}{c}\text { Guanosine } \\
\text { ( } \mu \mathrm{mol} / \mathrm{L})\end{array}$ & $\begin{array}{c}\text { dGuo } \\
(\mu \mathrm{mol} / \mathrm{L})\end{array}$ & $\begin{array}{c}\text { Inosine } \\
(\mu \mathrm{mol} / \mathrm{L})\end{array}$ & $\begin{array}{c}\text { dlno } \\
(\mu \mathrm{mol} / \mathrm{L})\end{array}$ & $\begin{array}{c}\text { PNP activity } \\
\text { (nmol/h/mg } \\
\text { [normal value, } \\
1684 \pm 561] \text { ) }\end{array}$ & $\begin{array}{c}\text { PNP } \\
\text { genotype } \\
\text { allele } 1\end{array}$ & $\begin{array}{c}\text { PNP } \\
\text { genotype } \\
\text { allele } 2\end{array}$ & $\begin{array}{c}\text { TREC } \\
\text { copies/ } / \mu \mathrm{L} \\
\text { (normal } \\
\text { value, }>10 \text { ) }\end{array}$ & $\begin{array}{c}\text { KREC } \\
\text { copies } / \mu \mathrm{L} \\
\text { (normal } \\
\text { value, }>8 \text { ) }\end{array}$ & Reference \\
\hline 1 & $11 \mathrm{mo}$ & 6.8 & 4.3 & 74.8 & 2.1 & 0.0 & c.287delT; V96fs $\dagger$ & c.548delA; E183fs ${ }^{\dagger}$ & & & Hershfield (personal data) \\
\hline 2 & $3 \mathrm{y}, 9 \mathrm{mo}$ & 6.0 & 4.3 & 50.9 & 0.9 & 2.5 & IVS3-18 G>A & IVS3-18 G>A & & & Markert et $\mathrm{al}^{16}$ \\
\hline 3 & $1 \mathrm{y}, 3 \mathrm{mo}$ & 5.5 & 6.3 & 454.6 & 2.2 & 12.1 & $\mathrm{P} 198 \mathrm{~L} \dagger$ & $\mathrm{P} 198 \mathrm{~L} \dagger$ & & & Hershfield (personal data) \\
\hline 4 & $13 \mathrm{y}$ & 2.9 & 2.8 & 34.3 & 1.3 & 18.4 & $\mathrm{~A} 117 \mathrm{~T}$ & A117T & & & Grunebaum et $\mathrm{al}^{17}$ \\
\hline 5 & $3 \mathrm{y}, 5 \mathrm{mo}$ & 1.7 & 0.2 & 27.1 & 2.3 & 683.0 & c.387_389 delCAT & c.387_389 delCAT & & & Markert et $\mathrm{al}^{16}$ \\
\hline 6 & $3 \mathrm{y}, 6 \mathrm{mo}$ & 2.9 & 3.2 & 31.5 & 1.7 & 24.5 & $\mathrm{R} 58 \mathrm{X}$ & E89X & & & Dalal et $\mathrm{al}^{18}$ \\
\hline 7 & $14 \mathrm{mo}$ & 2.6 & 0.2 & 283.3 & 5.5 & 0.0 & R58X & R58X & & & \\
\hline 8 & At birth & 3.7 & 7.4 & 192.6 & 17.3 & 16.0 & $\mathrm{~N} 243 \mathrm{~K} \dagger$ & $\mathrm{Y}_{249 \mathrm{~S}^{\dagger}}$ & 13.5 & 4.2 & Our data \\
\hline 9 & At birth & 7.1 & 3.4 & 50.2 & 1.3 & 0.0 & $\mathrm{D} 128 \mathrm{G}$ & D128G & 0 & 30 & Aust et al ${ }^{19}$ \\
\hline
\end{tabular}

*Per age reference values (Table II).

†Novel mutations.

\$Enzyme activity measured in induced pluripotent stem cell lines (normal value, 288-4018).

Our results support the inclusion of PNP-CID in routine newborn screening by using TMS. Detection of PNP metabolites by using TMS could be performed on the same DBSs collected at birth for routine screening tests and the method could be easily included in a population-based screening program because of its low cost and because it does not require extra equipment or extra time for operators already working in TMS screening programs. The absence of follow-up visits or blood examinations caused in screening based on other methods by the presence of falsepositive results would further increase the cost-effectiveness of this method. The inclusion of a disease into newborn screening panels depends on disease frequency and other factors and, among them, the sensitivity/specificity of the available screen tests, as well as their cost.

Even though PNP-deficient patients with early and delayed onset could be identified by using combined TREC and KREC screening programs, abnormal TREC levels, KREC levels, or both would require further functional analysis, whereas the TMS method would readily identify the enzymatic defect in both a specific and direct way.

Therefore, given the very low cost of the TMS tests, we suggest that PNP be included in the newborn screening panel in all newborn screening laboratories using TMS.

Clinical implications: TMS can measure PNP metabolite levels on newborn DBSs. It is a sensitive, specific, and inexpensive tool. PNP deficiency can be included in the TMS newborn screening panel.

\section{REFERENCES}

1. Notarangelo LD. Primary immunodeficiencies. J Allergy Clin Immunol 2010;125: $182-94$.

2. Kwan A, Church JA, Cowan MJ, Agarwal R, Kapoor N, Kohn DB, et al. Newborn screening for severe combined immunodeficiency and T-cell lymphopenia in California: results of the first 2 years. J Allergy Clin Immunol 2013;132:140-50.

3. Health Resources and Services Administration. Secretary's Advisory Committee on Heritable Disorders in Newborns and Children. Resolution from the 20th meeting, January 22, 2010; Washington, DC. Available at: http://www.hrsa.gov/ heritabledisorderscommittee/.

4. Markert ML. Purine nucleoside phosphorylase deficiency. Immunodefic Rev 1991; 3:45-81.

5. Somech R, Lev A, Grisaru-Soen G, Shiran SI, Simon AJ, Grunebaum E. Purine nucleoside phosphorylase deficiency presenting as severe combined immune deficiency. Immunol Res 2013;56:150-4.
6. Al-Saud B, Alsmadi O, Al-Muhsen S, Al-Ghonaium A, Al-Dhekri H, Arnaout R et al. A novel mutation in purine nucleoside phosphorylase in a child with normal uric acid levels. Clin Biochem 2009;42:1725-7.

7. Hershfield MS, Mitchell BS. Immunodeficiency diseases caused by adenosine deaminase deficiency and purine nucleoside phosphorylase deficiency. In: Scriver CR, Beaudet AL, Sly WS, Valle D, editors. The metabolic and molecular bases of inherited disease. 8th ed. New York: McGraw-Hill; 2001. pp. 2585-625.

8. Hershfield M. Combined immune deficiencies due to purine enzyme defects. In: Stiehm ER, Ochs HD, Winkelstein JA, editors. Immunologic disorders in infants and children. 5th ed. Philadelphia: Elsevier; 2004. pp. 480-504.

9. Azzari C, la Marca G, Resti M. Neonatal screening for severe combined immunodeficiency caused by an adenosine deaminase defect: a reliable and inexpensive method using tandem mass spectrometry. J Allergy Clin Immunol 2011;127: 1394-9.

10. la Marca G, Canessa C, Giocaliere E, Romano F, Duse M, Malvagia S, et al. Tandem mass spectrometry, but not T-cell receptor excision circle analysis, identifies newborns with late-onset adenosine deaminase deficiency. J Allergy Clin Immunol 2013;131:1604-10.

11. Puck JM. Neonatal screening for severe combined immunodeficiency (SCID). Curr Opin Pediatr 2011;23:667-73.

12. Borte S, von Döbeln U, Fasth A, Wang N, Janzi M, Winiarski J, et al. Neonatal screening for severe primary immunodeficiency diseases using high-throughput triplex real-time PCR. Blood 2012;119:2552-5.

13. la Marca G, Malvagia S, Casetta B, Pasquini E, Donati MA, Zammarchi E. Progress in expanded newborn screening for metabolic conditions by LC-MS/MS in Tuscany: update on methods to reduce false tests. J Inherit Metab Dis 2008;31: S395-404.

14. Arredondo-Vega FX, Kurtzberg J, Chaffee S, Santisteban I, Reisner E, Povey MS, et al. Paradoxical expression of adenosine deaminase in T cells cultured from a patient with adenosine deaminase deficiency and combine immunodeficiency. J Clin Invest 1990;86:444-52.

15. Arredondo-Vega FX, Santisteban I, Richard E, Bali P, Koleilat M, Loubser M, et al. Adenosine deaminase deficiency with mosaicism for a "second-site suppressor" of a splicing mutation: decline in revertant $\mathrm{T}$ lymphocytes during enzyme replacement therapy. Blood 2002;99:1005-13.

16. Markert ML, Finkel BD, McLaughlin TM, Watson TJ, Collard HR, McMahon CP, et al. Mutations in purine nucleoside phosphorylase deficiency. Hum Mutat 1997;9: 118-21.

17. Grunebaum E, Zhang J, Roifman CM. Novel mutations and hot-spots in patients with purine nucleoside phosphorylase deficiency. Nucleosides Nucleotides Nucleic Acids 2004;23:1411-5.

18. Dalal I, Grunebaum E, Cohen A, Roifman CM. Two novel mutations in a purine nucleoside phosphorylase (PNP)-deficient patient. Clin Genet 2001;59: 430-7.

19. Aust MR, Andrews LG, Barrett MJ, Norby-Slycord CJ, Markert ML. Molecular analysis of mutations in a patient with purine nucleoside phosphorylase deficiency. Am J Hum Genet 1992;51:763-72.

20. Speckmann C, Neumann C, Borte S, la Marca G, Sass JO, Wiech E, et al. Delayedonset adenosine deaminase deficiency: strategies for an early diagnosis. J Allergy Clin Immunol 2012;130:991-4. 


\section{METHODS TMS}

A Triple-Quad Mass Spectrometer (ABI SCIEX API 4000) equipped with the turbo ion-spray source and coupled to an analytic HPLC device was used for this study. The turbo ion-spray source operated under positive ion mode at a voltage of $5500 \mathrm{~V}$ and under a "turbo" gas flow of $10 \mathrm{~L} / \mathrm{min}$ of air heated to $500^{\circ} \mathrm{C}$ (nominal heating gun temperature).

Declustering potential, collision exit potential, and collision energy were automatically optimized for guanosine, inosine, dGuo, dIno, ${ }^{15} \mathrm{~N}_{5}$-Guo, ${ }^{15} \mathrm{~N}_{4}$-Ino, and ${ }^{2} \mathrm{H}_{2}$-dGuo by using the quantitation optimization option. The resulting declustering potential was $30 \mathrm{~V}$ for all of them. Optimal collision exit potential was $10 \mathrm{~V}$ for all metabolites, and labeled ISs and collision energies were found at $16 \mathrm{~V}$ for guanosine and ${ }^{15} \mathrm{~N}_{4}$-Guo; $14 \mathrm{~V}$ for inosine, dGuo, ${ }^{15} \mathrm{~N}_{4}$-Ino, and ${ }^{2} \mathrm{H}_{2}$-dGuo; and $13 \mathrm{~V}$ for dIno.

Mass spectrometric and TMS spectra were collected in continuous-flow mode by connecting the infusion pump directly to the turbo ion-spray source. Standard solutions of $1 \mathrm{ng} / \mathrm{mL}$ for guanosine, inosine, dGuo, dIno, ${ }^{15} \mathrm{~N}_{5}$-Guo,

${ }^{15} \mathrm{~N}_{4}$-Ino, and ${ }^{2} \mathrm{H}_{2}$-dGuo in an aqueous solution of $50 \%$ methanol containing $0.1 \%$ formic acid were infused at $7 \mu \mathrm{L} / \mathrm{min}$. From these experiments, the resulting ion-pair transitions for the quantitative experiment are as follows: $284.2>152.1$ and $289.2>157.1$ for guanosine and ${ }^{15} \mathrm{~N}_{5}$-Guo, respectively; $269.2>137.1$ and $273.2>141.1$ for inosine and ${ }^{15} \mathrm{~N}_{4}$-Ino, respectively; $268.2>152.1$ and $270.2>152.2$ for dGuo and ${ }^{2} \mathrm{H}_{2}$-dGuo, respectively; and $253.2>137.1$ for dIno. The extracted sample was injected into an electrospray triple-quadrupole tandem mass spectrometer with the aid of an automated liquid-handling device. Mass spectral data for the amino acids were acquired through a neutral loss scan of $46 \mathrm{Da}$; mass spectral data for the acylcarnitines were acquired through a precursor ion scan of $85 \mathrm{Th}$. Mass spectral data for purines, including adenosine and deoxyadenosine, and succinylacetone were acquired through multiple-reaction monitoring. The percentage of each analyte recovered was determined through comparison with an IS for each analyte.

\section{Method validation}

The method for individuating PNP metabolites on DBS samples has been patented (PCT EP2010/070517). The method provides quantitative analysis of different metabolites, among them guanosine, dGuo, inosine, and dIno. The method was validated by the use of calibrators at known concentration of PNP metabolites in terms of linearity, intraday and interday precision, and accuracy (Table E1). Calibrators containing ISs at $10 \mu \mathrm{mol} / \mathrm{L}$ were at concentrations of $0,1,10,50$, and $100 \mu \mathrm{mol} / \mathrm{L}$ for guanosine, dGuo, inosine, and dIno, respectively.

Reference standard blood spot calibrators were prepared at known concentrations of guanosine, dGuo, inosine, and dIno by using a pooled whole-blood sample from 3 healthy adult donors. A $20-\mu \mathrm{L}$ volume of spiked blood was dispensed onto filter paper cards and dried for at least 3 hours at room temperature.

A small disk ( $3.2 \mathrm{~mm})$ of a DBS was punched and deposited in a well of a microwell plate. The sample was extracted by dispensing $300 \mu \mathrm{L}$ of an extraction solution consisting of a mixture of methanol and aqueous solution of $3 \mathrm{mmol} / \mathrm{L}$ hydrate hydrazine at an approximate relative volume/volume ratio of $66.6 \%$ and $33.3 \%$, respectively. ISs for amino acids, acylcarnitines, succinylacetone, and purines (adenosine, deoxyadenosine, guanosine, dGuo, and inosine) were also present in the extract solution. The extracted sample was injected into an electrospray triple-quadrupole tandem mass spectrometer with the aid of an automated liquid-handling device. The percentage of each analyte recovered was determined through comparison with an IS for each analyte. Considering the unavailability of a commercially labeled IS of dIno, the quantitation of this metabolite was performed by means of external calibration with ${ }^{15} \mathrm{~N}_{4}$-Ino as the IS. The imprecision of the assay was determined by analyzing the samples described in Table E1. Each sample run consisted of 10 punches of each sample, which were processed and measured as previously described. The study included 10 such runs a day for a total of 10 days. With this information, the within-run, between-run/within-day, and between-day imprecision components were determined, from which the total imprecision was determined. The results of the imprecision analysis of guanosine, dGuo, inosine, and dIno are shown in Table E1.

\section{TREC and KREC analysis for patients 8 and 9}

DNA was eluted from a single 3.2-mm punch of the original Guthrie card of patient 8, as described previously, ${ }^{\mathrm{E} 1-\mathrm{E} 3}$ and DNA was extracted according to a previously described homemade procedure. ${ }^{\mathrm{E} 3}$ Quantitative triplex real-time quantitative PCR for TRECs, KRECs, and $\beta$-actin (ACTB) was performed on a ViiA7 real-time PCR system (Applied Biosystems, Foster City, Calif), as previously described, ${ }^{\mathrm{E} 3}$ for patient 7 . The quantitative PCR procedure was optimized based on custom reagents provided by Affymetrix (Santa Clara, Calif). TREC and KREC levels were normalized per microliter of blood, assuming that a single $3.2-\mathrm{mm}$ punch contains approximately $3 \mu \mathrm{L}$ of whole blood. Abnormal TREC/KREC screening results were defined as less than 8 TRECs $/ \mu \mathrm{L}$ and less than $6 \mathrm{KRECs} / \mu \mathrm{L}$ of dried blood based on cutoff values according to current prospective trials in Germany and Sweden.

As for patient 9, 3 punches from the regular screening card were obtained, and DNA was extracted according to the manufacturer's instructions for the QIAamp DNA Mini kit (Qiagen, Hilden, Germany). Two duplex real-time quantitative PCRs (TREC+ ACTB and KREC+ACTB) were performed on DBSs from patient 9 , as previously described. ${ }^{\mathrm{E} 1}$ The previously described method was used $^{\mathrm{E} 1}$ for both TREC and KREC measurements, with diagnostic cutoff scores at $10 \mathrm{TREC} / \mu \mathrm{L}$ and $8 \mathrm{KRECs} / \mu \mathrm{L}$. TREC and KREC analysis was always performed on the same samples used for TMS test.

Amplification of ACTB was used to assess the success of DNA extraction from the Guthrie cards.

\section{REFERENCES}

E1. la Marca G, Canessa C, Giocaliere E, Romano F, Duse M, Malvagia S, et al. Tandem mass spectrometry, but not T-cell receptor excision circle analysis, identifies newborns with late-onset adenosine deaminase deficiency. J Allergy Clin Immunol 2013:131:1604-10.

E2. Puck JM. Neonatal screening for severe combined immunodeficiency (SCID). Curr Opin Pediatr 2011;23:667-73.

E3. Borte S, von Döbeln U, Fasth A, Wang N, Janzi M, Winiarski J, et al. Neonatal screening for severe primary immunodeficiency diseases using high-throughput triplex real-time PCR. Blood 2012;119:2552-5. 


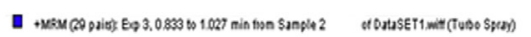

A

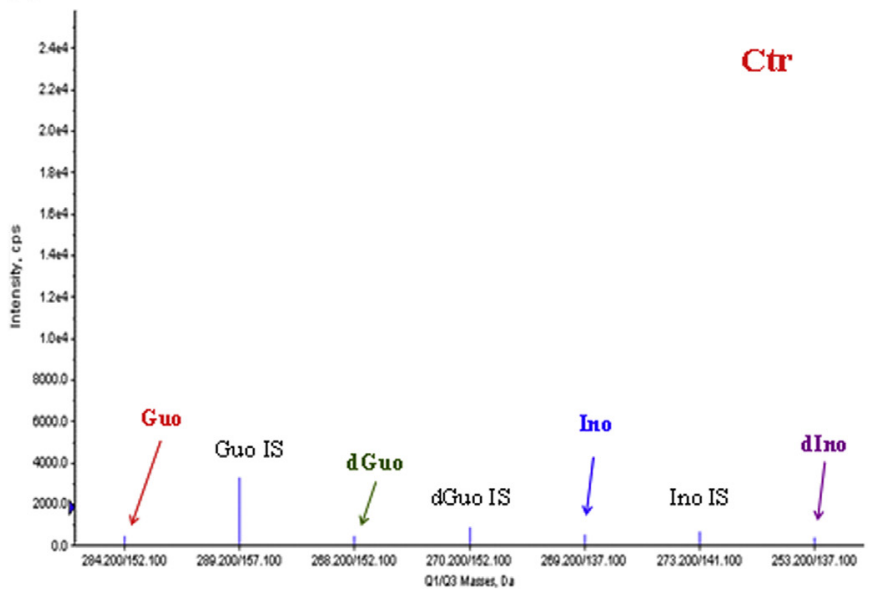

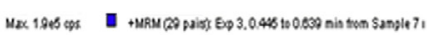

B

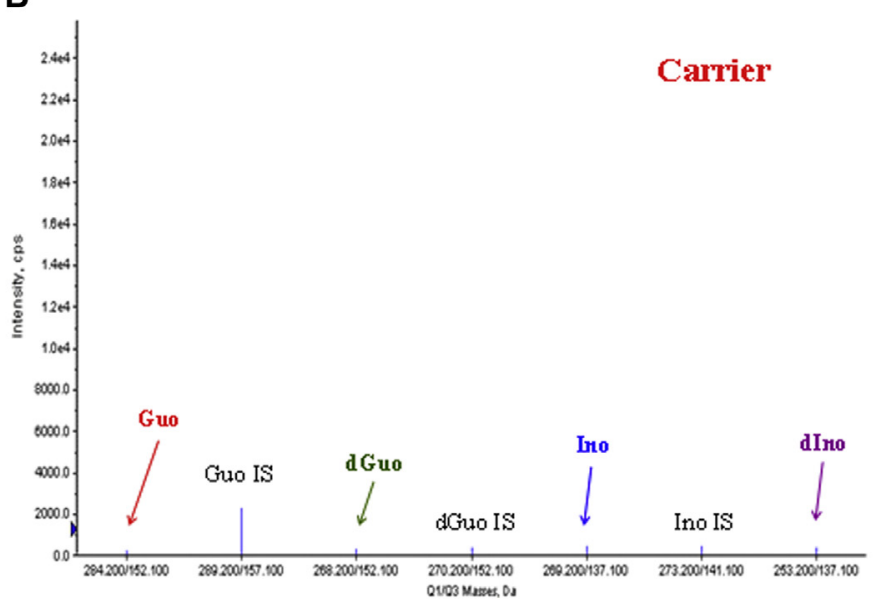

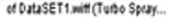

Mex $13.30 \mathrm{sen}$

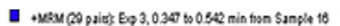

C

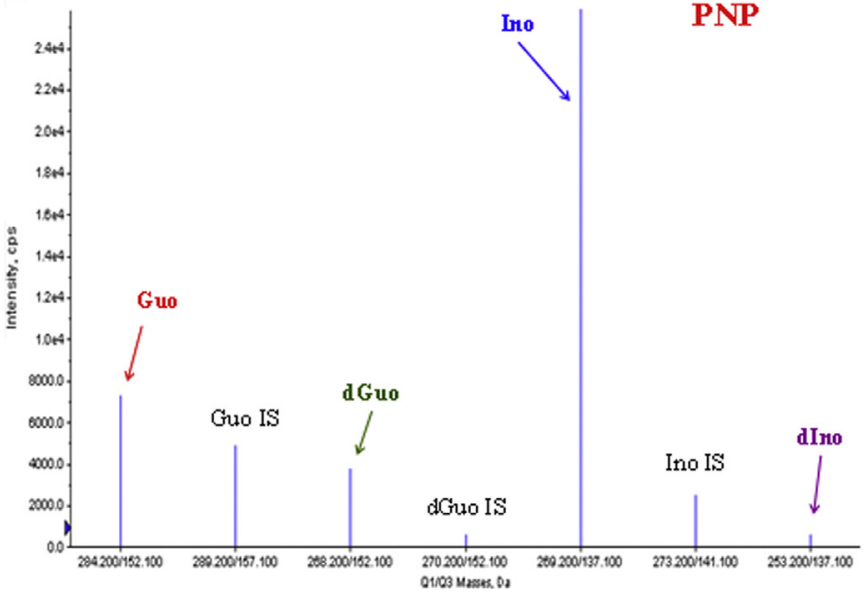

D

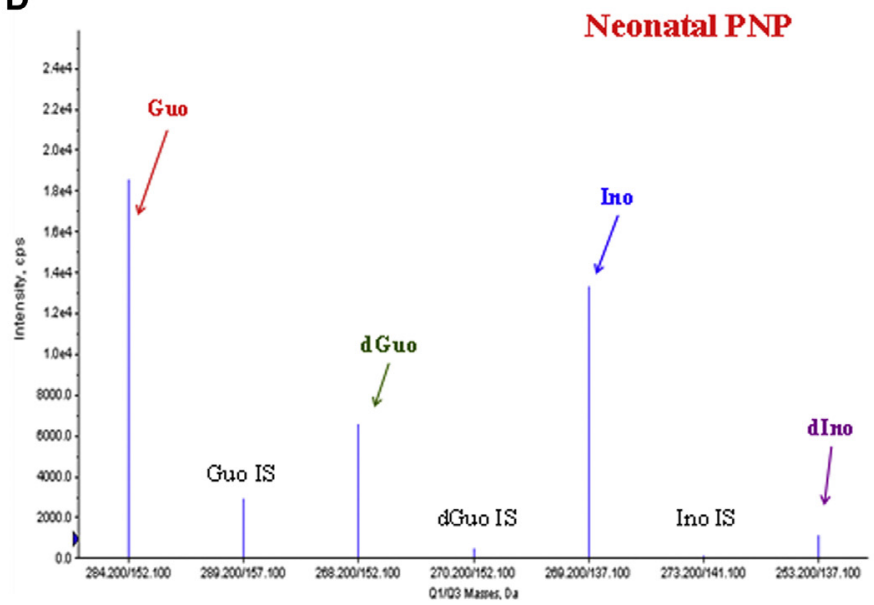

FIG E1. PNP marker profile in DBSs determined by using TMS analysis. A, Healthy control subject. B, PNP carrier. C, Patient 2 at the age of diagnosis. D, Retrospective analysis on neonatal card of patient 8 . Guo, Guanosine; Ino, inosine. 
159.e3 LA MARCA ET AL

TABLE E1. Intra- and interday imprecision and accuracy for PNP metabolites evaluated for method validation

\begin{tabular}{lrcccr}
\hline $\begin{array}{l}\text { Investigated } \\
\text { metabolite }\end{array}$ & $\begin{array}{c}\text { Spiking } \\
(\boldsymbol{\mu} \mathbf{m o l} / \mathbf{L})\end{array}$ & $\begin{array}{c}\text { Intraday } \\
\text { imprecision } \\
(\mathbf{\%}), \mathbf{n}=\mathbf{1 0}\end{array}$ & $\begin{array}{c}\text { Interday } \\
\text { imprecision } \\
(\mathbf{\%}), \mathbf{n}=\mathbf{1 0}\end{array}$ & $\begin{array}{c}\text { Average } \\
\text { readings } \\
(\boldsymbol{\mu} \mathbf{m o l} / \mathbf{L})\end{array}$ & $\begin{array}{c}\text { Accuracy } \\
(\mathbf{\%})\end{array}$ \\
\hline Guanosine & 1 & 4.1 & 1.9 & 0.8 & 82 \\
& 10 & 13.7 & 9.3 & 12.3 & 123 \\
& 50 & 11.5 & 6.4 & 57.5 & 115 \\
dGuo & 100 & 5.8 & 8.7 & 97.4 & 97 \\
& 1 & 6.3 & 4.8 & 1.3 & 125 \\
& 10 & 2.0 & 9.2 & 12.0 & 120 \\
Inosine & 50 & 2.7 & 15.2 & 49.4 & 99 \\
& 100 & 2.5 & 5.2 & 93.1 & 93 \\
& 1 & 3.2 & 4.7 & 1.0 & 99 \\
dIno & 10 & 3.4 & 14.6 & 10.5 & 105 \\
& 50 & 2.2 & 9.5 & 49.5 & 99 \\
& 100 & 1.9 & 13.5 & 96.3 & 96 \\
& 1 & 19.5 & 19.8 & 1.2 & 116 \\
& 10 & 5.0 & 7.3 & 11.4 & 114 \\
& 50 & 3.0 & 9.5 & 50.0 & 100 \\
& 100 & 1.9 & 8.0 & 101.0 & 101 \\
\hline
\end{tabular}

